\title{
Clinicoetiological and Biochemical Profile of Neonatal Convulsions
}

\author{
Yadav R K ${ }^{* 1}$, Sharma I K ${ }^{2}$, Kumar $D^{3}$, Shukla K M⿻ ${ }^{4}$, Jawwad $K^{5}$, Chaturvedi $V^{6}$ \\ ${ }^{1}$ Dr Rajesh Kumar Yadav, Associate Professor, ${ }^{2}$ Dr I K Sharma, Associate Professor, Department of Paediatrics, ${ }^{3} \mathrm{Dr}$ \\ Durgesh Kumar, Lecturer, Department of Paediatrics, ${ }^{4}$ Dr K M Shukla, Professor \& Head, Department of Paediatrics, \\ ${ }^{5}$ Dr Kalbe Jawwad, Professor \& Head, Department of Biochemistry, ${ }^{6}$ Dr Vineet Chaturvedi, Associate Professor \\ Department of Pathology. All are affiliated with UPRIMS \& R, Saifai, Etawah, UP, India
}

*Address for correspondence: Dr Rajesh Kumar Yadav, department of pediatrics, UPRIMS \& R Saifai, Etawah UP. E-mail ID: rajeshsaifai@gmail.com

\begin{abstract}
Introduction: Neonatal seizures may present in $1-2 \%$ of term to almost $20 \%$ in preterm infants. Hypoxic ischemic encephalopathy, sepsis and bacterial meningitis are common causes. Biochemical abnormality and intra cranial bleed is also quiet common in this age. Aim of our study was to observe clinical presentation, to find common etiological factors and analysis of associated biochemical abnormality in newborn presented with neonatal convulsions in our hospital. Methods: Prospective hospital based observational study. From January 2014 to December 2014, total 168 newborns admitted with convulsions or developed convulsions during hospitalization, 154 were included in the study. After taking complete history and appropriate physical examination, blood sample was collected before instituting specific therapy. Investigations advised to all were complete blood count, sepsis screen, blood sugar, serum electrolytes. CSF study, serum bilirubin, USG cranium, CT scan or MRI was also ordered when indicated. Results: Cumulative frequency of neonatal convulsion in our study was $5.52 \%$. Perinatal asphyxia was most common etiological factor in term or post term babies and presentation of seizures was early, intra cranial bleed and metabolic abnormality was common cause of convulsions in premature babies and presentation of seizure was somewhat late. Subtle seizure was most common type of fits followed by clonic type of seizures. Hypocalcemia was most common biochemical derangement. Conclusion: hypoxic ischemic encephalopathy was most common etiology of neonatal convulsions, presented with convulsions within 72 hours of life and subtle seizure and focal clonic or multifocal clonic was most common seizure type.
\end{abstract}

Keywords: Biochemical Disturbances, Etiological, Neonatal Seizure, Perinatal Asphyxia

\section{Introduction}

Seizures in neonatal period are more common than any other age because of immature brain functions and improper myelination, even more common in preterm babies than term babies. Incidence of seizures ranges from 2.8/1000 in term appropriate for gestational age to $57.5 / 1000$ in pre term very low birth weight babies (VLBW) [1]. A seizure is defined as paroxysmal electrical discharge from brain which may manifest as motor, sensory, behavioural or autonomic dysfunctions [1]. It results from excessive synchronous electrical discharge, due to depolarization of neurons because of inward movement of sodium ions and instead of hyperpolarization, depolarization of neuron on inward

Manuscript received: $30^{\text {th }}$ Sept 2015

Reviewed: $10^{\text {th }}$ Oct 2015

Author Corrected: $18^{\text {th }}$ Oct 2015

Accepted for Publication: $2^{\text {nd }}$ Nov 2015 movement of chloride ion in immature newborn [2].

Most seizures in the newborn are symptomatic of a specific aetiology and with diagnostic advances, aetiology is easily identifiable. Neonatal seizures are different in manifestation as well as response to treatment due to immature development state of newborn brain and different etiologies.

Seizures in neonatal period can be subtle, focal clonic, multi focal clonic, tonic spasm and myoclonic. Spasm, focal clonic or tonic and generalized myoclonic seizures are associated with electrographic discharges, whereas the subtle, generalized tonic and other myoclonic seizures are not associated with EEG discharges [1]. Common causes of convulsions in newborn are hypoxic ischemic encephalopathy, cerebral infarction and stroke, intra cranial hemorrhage, intra cranial infections 
metabolic disturbances and undetermined, etc [3]. Tonic seizure and myoclonic seizures were associated with unfavourable outcome and found in infants with hypoxic ischemic encephalopathy and intra cranial hemorrhage. Most common Biochemical abnormality associated with neonatal convulsion is hypocalcemia, hypoglycaemia, hypomagnesemia and hyponatremia. Hypoglycaemia in $50 \%$ cases associated with unfavourable outcome [3].

Most of data on neonatal convulsions are from western literature. Incidence of neonatal convulsions and its aetiology in developing world may be different from western regions. Our aim is to identify the aetiology, its clinical presentation and biochemical changes in cases of neonatal convulsions in our region.

\section{Materials and Methods}

This was a prospective observational study conducted at the level II newborn care unit of a teaching hospital located in north India. All the term and pre term neonates from birth to 28 days of life, both intra mural and extramural, admitted in the department of Pediatrics, from Jan 2014 to December 2014 with clinically apparent convulsions, history of convulsions or who develop convulsions during hospitalization was enrolled in the study. Clinical criteria for diagnosing neonatal convulsions were [3], (a) focal, multifocal, or generalised clonic movement (b) tonic posturing with or without abnormal gaze (c) subtle seizures and spontaneous paroxysmal repetitive motor or autonomic phenomenon like lip smacking, chewing, paddling, swimming, cyclic movements or respiratory abnormalities.

Doctors and nursing staff posted in the neonatal care unit were trained properly to identify, classify and diagnose neonatal convulsions and to differentiate between convulsions and non epileptic movements [3]. Infants having apparent body movements consistent with non epileptic movements were excluded from the study. Ethical committee of the Institute has approved this study. Written informed consent is taken from parent or care giver prior to enrolment of neonate for the study. After proper management of airway breathing, circulation according to standard guidelines [3], baseline characteristics of all the babies were recorded on the prescribed proforma which includes name, age, sex, address weight, length, head circumference, gestational age, which is determined from parent by LMP or ultrasound study of fetus before birth or by new Ballard score of baby [4].

After stabilizing the baby and insertion of IV canula, blood sample was taken for blood sugar, serum calcium, serum electrolytes and other blood tests. Hypoglycaemia was defined as serum glucose level less than $45 \mathrm{mg} / \mathrm{dl}$ and hypocalcemia was defined as ionized calcium less than $1 \mathrm{mmol} / 1$ in term neonates and less than $1.2 \mathrm{mmol} / \mathrm{l}$, in pre term babies [3]. After stabilizing and correcting metabolic abnormality if present, newborns were subjected to antiepileptic as per standard guidelines [5]. After initial management, doctor on duty took detailed history of seizure, its age of onset, duration and character of seizure. History of trauma, medication and other relevant history were also recorded on pre structured proforma. History of maternal illness, antenatal, natal and birth history, place of delivery, mode of delivery Apgar score and any relevant event just after delivery, fever, or hypothermia, feeding details were recorded. History consistent with perinatal asphyxia was also sought for. Hypoxic ischemic encephalopathy defined and staging is done on the basis of Levene classification [6]. Convulsions were classified on the basis of criteria by Volpe [7]. Thorough physical examination including vitals, general examination systemic examination, neonatal reflexes, ocular examination, search for organomegaly and Icterus were done and recorded.

Routine investigations like complete blood count, Peripheral blood smear, band cells, serum electrolytes. Sepsis screen and Cerebral Spinal Fluid analysis, in case of suspected sepsis were carried out. SGA Babies presented with cataract, rashes, microcephaly, chorioretinitis, icterus and hepatosplenomegaly were subjected to TORCH study. Urine analysis, Kidney function test, Liver function test, EEG, Neurosonogram, CT Scan or MRI brain was done when required.

Criteria for the various other metabolic / biochemical derangement are defined as follows [8]. Hyponatremia was serum sodium < $130 \mathrm{meq} / \mathrm{L}$, Hypernatremia was serum sodium $>150 \mathrm{meq} / \mathrm{L}$ and normal range $130-150$ meq/L. Hypomagnesaemia defined as serum magnesium level $<1.5 \mathrm{mg} / \mathrm{dl}$, and normal range is $1.5-$ $1.8 \mathrm{mg} / \mathrm{dl}$, measured by atomic absorption spectrophotometry. Hypokalemia was serum potassium less than $3.5 \mathrm{meq} / \mathrm{l}$ and normal range is $3.5-5.5 \mathrm{meq} / \mathrm{l}$. Benign fifth day fits occur between 2-5 days of life in otherwise healthy newborns with normal EEG and spontaneous recovery. 
Sample size - Previous studies on neonatal seizures were done by taking sample of 60 to 100 cases $[8,9,10$, $11,15]$. Taking account of the incidence reported in various studies $1-4 \%$, the estimated sample size should be more than 65 . We conducted the study from January
2014 to December 2014 and admitted 168 neonates with convulsions, out which 14 were excluded for various reasons so that a total 154 babies were recruited for the study. Fig 1 shows flow chart of cases included-

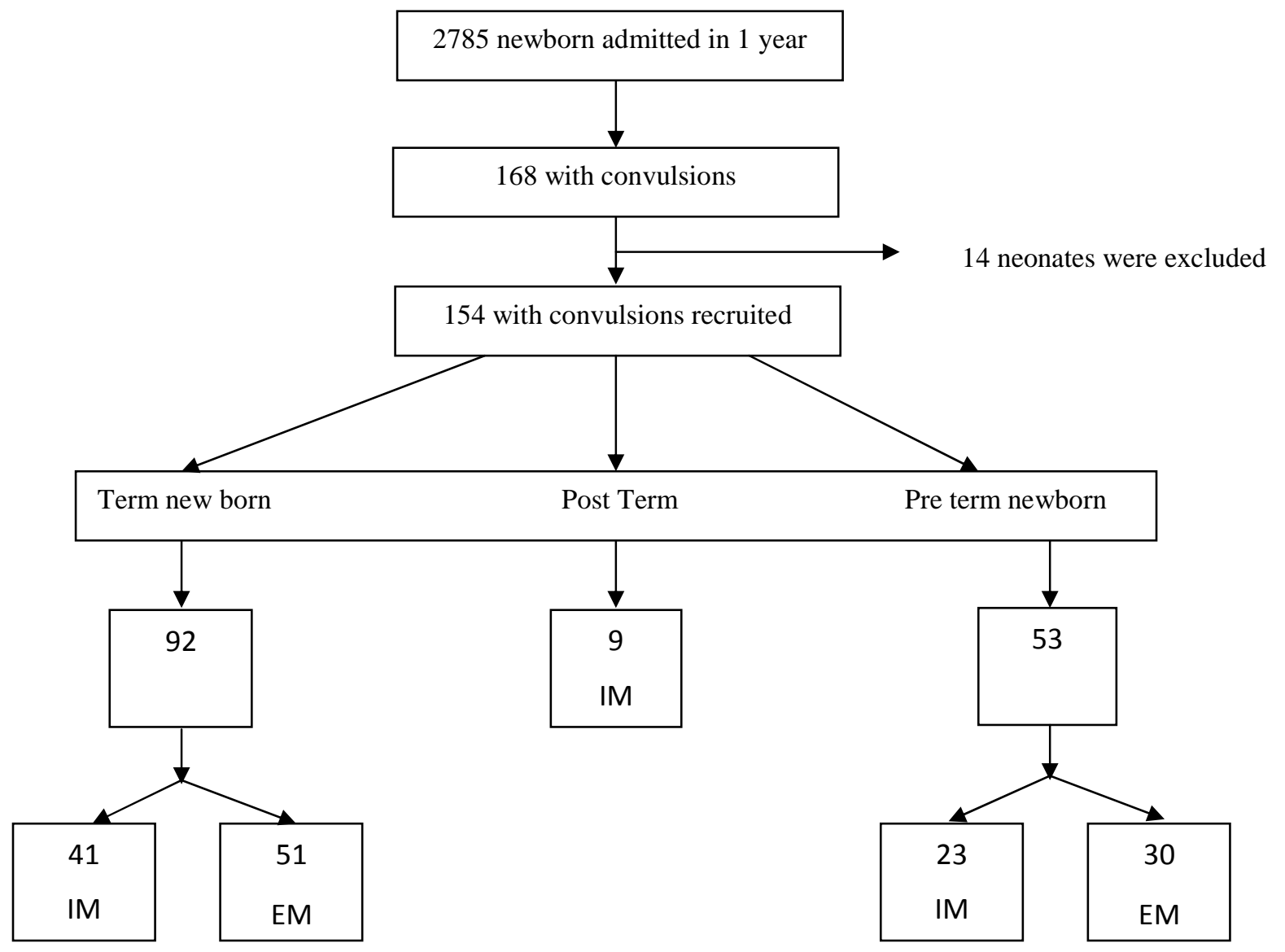

Fig1-Flow chart of study population

IM - intramural -baby delivered in our hospital, EM- extramural -baby delivered other than our hospital.

Data was recorded on MS Excel sheets and analysed by statistical package for social sciences (SPSS. version16). Data was described as mean \pm SE and \%age. P value less than 0.05 was considered significant.

\section{Results}

During the study period, 6527 babies were delivered in our Institute out of which $58 \%$ babies were delivered normally by vaginal route, $27 \%$ babies were delivered by LSCS and $15 \%$ babies were born with assistance or instrumentation. A total 2785 newborns were admitted in the pediatrics department for various causes, both delivered in our hospital (intramural 1380) as well as delivered outside (extramural 1405). 168 newborns presented with convulsions or developed during hospitalization. Cumulative frequency of convulsions in our study was about $5.52 \%$ (4.56\% in intramural group and $6.47 \%$ in extramural group).

Among the total, male babies were predominant and accounted for $62.33 \%$. Out of 154 neonates, Pre term babies were 53 (34.41\%), term babies were $92(59.74 \%)$ and post term baby were $9(5.84 \%)$. and 89\% babies were AGA, 7\% neonates were SGA and approx. 3\% were LGA. Among the premature babies, $61 \%$ were male. The mean weight and length of babies was $2487 \mathrm{~g} \pm 645$ and $47.34 \mathrm{~cm} \pm 2.86$, respectively. Delivery in 73 babies $(47.40 \%)$ were intramural while in 81 babies $(52.50 \%)$ were extramural. Table 1 shows baseline characteristics of admitted cases. 
Table 1: Characteristics of newborn with convulsions

\begin{tabular}{|l|l|l|}
\hline Particulars & Number & \% \\
\hline GA - & & \\
Pre Term & 53 & 34.41 \\
Term & 92 & 59.74 \\
Post Term & 9 & 5.84 \\
\hline Wt. & & \\
AGA & 137 & 88.96 \\
LGA & 5 & 3.25 \\
SGA & 12 & 7.79 \\
\hline Intra mural delivery & 73 & 47.40 \\
Extra mural delivery & 81 & 52.50 \\
\hline Gender & & \\
Male & 96 & 62.33 \\
Female & 58 & 37.66 \\
\hline
\end{tabular}

Common presentation at the time of admission was abnormal body movements or abnormal posturing (fits), refusal to feed, fever or hypothermia, cyanosis and icterus. Onset of seizure within 24 hours of delivery was found in $31 \%$ of infants, while convulsions within 48 hours of delivery were seen in 52\% babies. Convulsions before 72 hours of life were seen in $67 \%$ neonates, while from $4^{\text {th }}$ day of life to $7^{\text {th }}$ day, $11 \%$ infants presented with convulsions. $14 \%$ neonates developed seizures during $1^{\text {st }}$ and $2^{\text {nd }}$ week of life. From $2^{\text {nd }}$ to $4^{\text {th }}$ week of life, approximately $8 \%$ neonates presented with seizures.

Table 2: Clinical and etiological characteristics

\begin{tabular}{|l|l|l|}
\hline Clinical presentation & Number & $\%$ \\
\hline Convulsions & 154 & 100 \\
\hline Refusal to feed/ difficulty in feeding & 80 & 52 \\
\hline Fever or hypothermia & 63 & 41 \\
\hline Cyanosis & 55 & 36 \\
\hline Icterus & 37 & 24 \\
\hline Causes of seizure & Number & $\%$ \\
\hline Birth Asphyxia & $\mathbf{6 7}$ & $\mathbf{4 3 . 5 2}$ \\
1. HIE Gr I & 6 & 3.89 \\
2. HIE Gr II & 41 & 26.62 \\
$3 . \quad$ HIE Gr III & 20 & 13 \\
\hline Intra cranial bleed & 25 & 16.23 \\
\hline Metabolic & 24 & 15.60 \\
\hline Meningitis/ septicaemia & 17 & 11 \\
\hline Kernicterus & 5 & 3.25 \\
\hline Neonatal Tetanus & 4 & 2.60 \\
\hline Miscellaneous* & 12 & 7.8 \\
\hline
\end{tabular}

Miscellaneous* includes undetermined, structural anomaly IEM etc.

Most common etiology in our study was birth asphyxia and found in 67 cases (43\%) of neonatal convulsions and affecting term, pre term and even post term babies. Intra cranial bleed was etiology for seizures in 25 cases (16.25\%). Primary metabolic derangement was found in 24 cases $(15.60 \%)$, which manifest in the order of hypocalcaemia in 10 babies followed by hypoglycaemia in 8 babies, hypomagnesaemia in 4 and Hyponatremia in 2 babies. Primary Metabolic derangement as cause of neonatal convulsion was found in $16 \%$ in our study which was also comparable to previous studies. Neonates with meningitis or septicaemia constituted $11 \%$ cases. Kernicterus and neonatal tetanus were found in 5 and 4 newborns respectively. 12 babies $(7.8 \%)$ were included in the undetermined group like benign $5^{\text {th }}$ day fits, in born error of metabolism, structural anomaly etc. 
In our study, it became clear that early onset neonatal convulsions are more commonly associated with clinical aetiology, like hypoxic ischemic encephalopathy, meningitis, etc, and late onset neonatal convulsions are found more frequently in biochemical or metabolic derangements. Intra cranial bleed was most commonly associated with pre term babies and presented as late onset convulsion. Hypomagnesaemia was found in 4 newborns, presented as convulsions with late onset hypocalcaemia not responding to specific therapy. Primary hypocalcemia was also a common cause of late onset neonatal convulsions. Hyponatremia was present in 2 babies, one associated with meningitis and other with acute kidney injury because of asphyxia. Most common secondary metabolic derangement found in our study was hypocalcaemia and hypoglycaemia. Hypocalcaemia was mainly associated with hypoxic ischemic encephalopathy, prematurity and faulty feeding, while hypoglycaemia was mostly associated with prematurity, infant of diabetic mother, sepsis, hypothermia, asphyxia and faulty feeding methods etc.

Table 3: Type of neonatal convulsions

\begin{tabular}{|l|l|l|}
\hline Types & Number & \% \\
\hline Subtle seizures & 63 & 40.90 \\
\hline Clonic seizures & 52 & 33.76 \\
\hline Tonic seizures & 37 & 24 \\
\hline Myoclonic & 2 & 1.3 \\
\hline
\end{tabular}

Most common type of seizure was subtle type which presented in 63 newborns and constituted $41 \%$ approximately. The clonic seizure was present in 52 babies and accounted for approx. 34\%. Tonic convulsions were present in 37 babies and accounted for $24 \%$ and myoclonic spasm occurred in only 2 babies. Most of cases of birth asphyxia were presented with focal or multi focal clonic seizures or subtle seizures, while cases of intra cranial bleed or meningitis were presented with subtle seizures. Pre term babies were presented mostly with subtle seizures or focal tonic or focal clonic seizures

Primary hypocalcaemia and hypoglycaemia was mostly presented with tonic convulsions. Among the hypoxic ischemic encephalopathy, HIE II was most commonly associated with neonatal convulsion followed by HIE stage III. 6 babies in HIE stage I also presented with convulsions. Out of 154 babies, 17 died during hospitalization in our set up, 6 babies went LAMA and 13 lost to follow up. 118 babies were followed periodically.

\section{Discussion}

Seizures are most common neurological disorders in newborn period and incidence reported varies from 2.8/1000 live birth in term AGA, newborn to 57.5/1000 live birth in very low birth weight babies [1]. Onset of seizure and its duration and biochemical derangement can be correlated with the aetiology and prognosis of neonatal seizures. Early diagnosis and appropriate intervention can prevent from further deterioration of brain functions. This study demonstrates that frequency of convulsions in our study was approx $5.52 \%$ of the admitted newborns over a 1 year period in our ward, although the frequency of neonatal seizures may range from $1 \%$ to $10 \%$ in various reported studies. Our set up is located in a rural area of north India, catering mainly to rural population with improper health facilities. So chances are more that we get newborns in a compromised state. In our study NS was more common in male babies $62.33 \%$. On the gestational period basis, full term babies presented with seizures were $60 \%$, preterm babies were $34 \%$ and post term babies were $6 \%$ approximately. These findings were in concordance with several previous studies [8-11].

We found in our study, subtle seizure in 63 cases (41\%) which were most commonly associated with birth asphyxia and pre term babies, followed by clonic seizure in $34 \%$ babies with asphyxia or prematurity and intra cranial bleed were the leading causes. Tonic seizures were present in $24 \%$ cases and were associated with meningitis, intra cranial bleed and birth asphyxia. Aziz A et al [10], reported clonic convulsions are more common while Taksande et al [12], reported subtle seizures as the commonest and occurring in $50 \%$ cases. $52 \%$ neonates presented with early onset neonatal convulsions ie convulsions occurring within 48 hours of birth. Within the $1^{\text {st }}$ week, convulsions were present in $78 \%$ babies. Newborns presented with convulsions during $1^{\text {st }}$ and $2^{\text {nd }}$ week were about $14 \%$ and during 2 nd and $4^{\text {th }}$ weeks of life were approximately $8 \%$ only. This presentation is consistent with earlier studies [13-15]. 
Most common cause of neonatal convulsions was birth asphyxia or its complication, and in our study it was found in $43 \%$ cases. Frequency of birth asphyxia as a cause of neonatal convulsions, reported by previous authors like Sood A et al [16], Kumar A et al [17], and Aziz A et al [10], were $45.71 \%, 48.2 \%$ and $44 \%$, respectively and comparable to our data. Term babies were commonly associated with asphyxia, while premature babies with convulsions were having perinatal asphyxia, hypoglycaemia, and hypocalcemia or intra cranial bleed. Intra cranial bleed in a premature newborn was mostly in intra ventricular or periventricular while extra dural hemmorhage was mostly found in term neonates. Intra cranial bleed as a cause of neonatal convulsions was found in $16 \%$ cases, which is slightly higher than previous reported data like Bushra A et al [18], reported 9.5\% and Aziz A et al [10], reported $13 \%$ of cases while in our study intra cranial bleed accounted for $16 \%$ cases, which may be because of more premature babies in our study. Rose et al [19], and Scher et al [20], reported higher incidence of intra cranial bleed in pre term babies.

Primary metabolic abnormality was found in $16 \%$ babies with neonatal convulsions, of which $63 \%$ was because of hypocalcaemia and $30 \%$ because of hypoglycaemia. Late hypocalcemia not relieved by specific therapy was associated with hypomagnesaemia in 4 cases. These findings were comparable to the previous studies. Neonatal infections / meningitis were present in $11 \%$ cases in our study. Among the neonatal infection, early onset neonatal infection was more common and present in $58 \%$ cases. Rabindran et al [15], reported meningitis as a cause of NS in $7.69 \%$ cases where as Legido A et al [21], reported 17.2\%, while Aziz A et al [10], reported $22 \%$ incidence of neonatal infection in neonatal seizures. Our findings are comparable and more likely confounded by hygiene.

\section{Conclusion}

Most common presentation of neonatal convulsions was subtle seizures followed by focal or multi focal clonic and tonic type. Convulsion presented in $52 \%$ cases in first 48 hours and $78 \%$ within first week. The most common aetiology is found to be hypoxic ischemic encephalopathy followed by intra cranial bleed, primary metabolic abnormality and meningitis or neonatal infections. Transient biochemical derangement was commonly associated with birth asphyxia, meningitis etc and most common abnormality was hypocalcaemia and hypoglycaemia.
Acknowledgement - Authors are thankful to the newborn's parents for giving consent for the study.

Conflict of interest: None declared.

Funding: Nil, Permission from IRB: Yes

\section{References}

1. Mikati MA, Kliegman RM, Behrman RE, Stanton BF. Seizures in childhood; Nelson textbook of Paediatrics, 19th edition. Philadelphia. 2011: WB Saunders [586]: 2013-2037.

2. Nanavati RN, Parthasarthy A, Menon PSN, Gupta P, Nair MKC. Neonatal seizures; IAP textbook of Paediatrics, $5^{\text {th }}$ edition. Newdelhi. 2013: Jaypee [2.9]: 4751.

3. Sankar MJ, Agarwal R, Aggarwal R, Deorari AK, Paul VK. Seizures in the newborn. Indian J Pediatr. 2008 Feb;75(2):149-55.

4. Ballard JL, Khoury JC, Wedig K, Wang L, EilersWalsman BL, Lipp R. New Ballard Score, expanded to include extremely premature infants. J Pediatr. 1991 Sep;119(3):417-23.

5. Plessis Ade J. Du, Cloherty John P, Eichenwald Eric C, Stark Ann R. Neonatal seizures; Manual of Neonatal care, $6^{\text {th }}$ edition. New delhi. 2008: [27A]: $483-498$.

6. Levene MI. The asphyxiated newborn infant. In Levene MI, Lilford RJ, ed. Fetal and neonatal neurology and neurosurgery. Churchill Livingstone, Edinburgh $1995 ; 405-26$

7. Volpe JJ. Neonatal seizures. Neurology of the newborn. Philadelphia, PA: WB Saunders, 2001;178-214.

8. Kumar A, Gupta V, Kachhawaha JS, Singla PN. Biochemical abnormalities in neonatal seizures. Indian Pediatr. 1995 Apr;32(4):424-8.

9. Mizrah EM. Neonatal seizures and neonatal epileptic syndromes. Neurol Clin. 2001 May;19(2):427-63.

10. Aziz A, Gattoo I, Aziz M, Rasool G. Clinical and etiological profile of neonatal seizures: a tertiary care hospital based study. Int J Res Med Sci 2015; 3:21982203.

11. Najeeb S, Qureshi AM, Anis-ur-Rehman, Ahmad F, Shah S, Khan AY, Siddiqui TS. Aetiology and types of 
neonatal seizures presenting at Ayub Teaching Hospital Abbottabad. J Ayub Med Coll Abbottabad. 2012 JanMar;24(1):33-7.

12. Taksande AM, Krishna V, Manish Jain, Mahaveer L. Clinico-biochemical profile of neonatal seizures. Paed Oncall Journal 2005 October;2(10).

13. Plouin P, Kaminska A. Neonatal seizures. Handb Clin Neurol. 2013;111:467-76. doi: 10.1016/B978-0-44452891-9.00051-8.

14. Kumar A, Gupta A, Talukdar B. Clinico-etiological and EEG profile of neonatal seizures. Indian $J$ Pediatr. 2007 Jan;74(1):33-7.

15. Rabindran, Hemant Parakh, Ramesh J K, Prashant Reddy. Phenobarbitone for the Management of Neonatal Seizures - A Single Center Study. Int J Med Res Rev 2015;3(1):63-71.
16. Sood A, Grover N, Sharma R. Biochemical abnormalities in neonatal seizures. Indian J Pediatr. 2003 Mar;70(3):221-4.

17. Kumar A, Gupta A, Talukdar B. Clinicoetiological and EEG profile of neonatal seizures. Indian J Pediatr. 2007 Jan;74(1):33-7.

18. Malik BA, Butt MA, Shamoon M, Tehseen Z, Fatima A, Hashmat N. Seizures etiology in the newborn period. J Coll Physicians Surg Pak. 2005 Dec;15(12):786-90.

19. Rose AL, Lombroso CT. A study of clinical, pathological, and electroencephalographic features in 137 full-term babies with a long-term follow-up. Pediatrics. 1970 Mar;45(3):404-25.

20. Scher MS. Controversies regarding neonatal seizure recognition. Epileptic Disord. 2002 Jun;4(2):139-58.

21. Legido A, Clancy RR, Berman PH. Neurologic outcome after electroencephalographically proven neonatal seizures. Pediatrics. 1991 Sep;88(3):583-96.

\section{How to cite this article?}

Yadav R K, Sharma I K, Kumar D, Shukla K M, Jawwad K, Chaturvedi V. Clinicoetiological and Biochemical Profile of Neonatal Convulsions. Int J Med Res Rev 2015;3(9):1057-1063. doi: 10.17511/ijmrr.2015.19.193. 